\title{
Evidence-based consensus on opportunistic infections in inflammatory bowel disease (republication)
}

\author{
Inflammatory Bowel Disease Group, Chinese Society of Gastroenterology, Chinese Medical Association
}

Inflammatory bowel disease (IBD) patients are a high-risk population for opportunistic infections. The IBD group of the Chinese Society of Gastroenterology of the Chinese Medical Association organized an expert group to discuss and develop this consensus opinion. This consensus opinion referenced clinical study results from China and other countries to provide guidance for clinical practices. Eight major topics, including cytomegalovirus infection, Epstein-Barr virus infection, viral hepatitis, bacterial infection, Mycobacterium tuberculosis infection, fungal infection, parasitic infection, and vaccines were introduced in this article. (Intest Res 2018;16:178-193)

Key Words: Inflammatory bowel disease; Opportunistic infections; Consensus

\section{INTRODUCTION}

Inflammatory bowel disease (IBD) is a chronic nonspecific intestinal disease mainly including CD and UC. The chronic, protracted course of IBD severely affects the patient's quality of life. The treatment of IBD has gone through 3 phases. Glucocorticoids were introduced in the 1950s, immunosuppressive agents began to be used in the 1960s, and therapeutics is now in the era of biological agents. With the progression and continuous standardization of its treatment, treatment efficacy for IBD has made great progress, resulted in significantly decreased complications and mortality in the patients. However, monotherapy or combination therapy with these drugs will change patient's immune status, which may bring corresponding issues, particularly an increased risk of opportunistic infection. ${ }^{1}$

Opportunistic infection refers to micro-organisms that have limited or no pathogenic capacity in healthy human

Received March 14, 2018. Revised April 8, 2018.

Accepted April 16, 2018.

Correspondence to Jiaming Qian, Department of Gastroenterology, Peking Union Medical College Hospital, Peking Union Medical College, Chinese Academy of Medical Sciences, No.1 Shuaifuyuan, Beijing 100730, China. Tel: +86-10-65295019, Fax: +86-10-6529-5019, E-mail: qianjiaming1957@126.com

This article is based on a study first reported in the Journal of Digestive

Disease 2018;19(2);54-65. bodies but cause diseases or induce infections when the immune system is compromised by other diseases (such as acquired immunodeficiency syndrome) or drug medications (such as immunosuppressive agents). IBD patients are high-risk populations for opportunistic infections. First, the disease itself can cause a reduced nutritional status in IBD patients. Second, the application of glucocorticoids, immunosuppressive agents, and biological agents can severely inhibit patient's immunity. Therefore, patients with IBD are susceptible to opportunistic infections, which requires much attention in clinical setting. ${ }^{2,3}$ The prevalence of various infectious diseases are relatively higher in China compared with those in the Western countries. Positive prevention, early diagnosis and timely control of opportunistic infections are the premise for improving the prognosis of IBD patients in China. ${ }^{4}$ This consensus referenced the results of clinical researches from both China and abroad, in combination with the characteristics of Chinese IBD patients, to provide guidance for clinical practices.

The IBD group of the Chinese Society of Gastroenterology, Chinese Medical Association organized the experts from the aspects of IBD, infection, clinical epidemiology and evidence-based medicine to establish this consensus using the Delphi method. Our IBD experts were divided into 7 research groups to perform literature retrieval, screen-

๑ Copyright 2018. Korean Association for the Study of Intestinal Diseases. All rights reserved.

This is an Open Access article distributed under the terms of the Creative Commons Attribution Non-Commercial License (http://creativecommons.org/licenses/by-nc/4.0)

which permits unrestricted non-commercial use, distribution, and reproduction in any medium, provided the original work is properly cited. 
ing and assessments to confirm the patients-interventioncomparison-outcome issues that needed to be solved in the consensus. Three rounds of voting (with a total of 58 experts participating in it) were conducted through electronic mails, and the ballots were counted by a third party. Subsequently, in-person voting using a voting machine was conducted with a total of 24 experts. After 2 expert seminars, the draft was discussed, amended, and passed in the plenary meeting of the IBD group in China. After being reviewed and finalized by experts in the infection and clinical epidemiology, this consensus was finally established.

The 3 recommendation levels of this consensus are as follows: level A, where the rating standard a was passed by 3 out of 4 or higher voting; level B, where the rating standard $a+b$ was passed by 3 out of 4 or higher voting; and level C, where the above-mentioned indicators were not reached and were deleted. The rating standards were: (1) total approval (indispensable, minimal requirement); (2) partial approval and recommendation (should be done, but did not reach the essential level); (3) depended on the conditions; and (4) disapproval (deleted, unreasonable, unnecessary, incompatible with national conditions, inoperatable, not assessable, and no need to include this item in the consensus).

Eight major topics, including cytomegalovirus (CMV) infection, Epstein-Barr virus (EBV) infection, viral hepatitis, bacterial infection, Mycobacterium tuberculosis infection, fungal infection, parasitic infection, and vaccines were discussed in this consensus (a total of 33 indicators) (Table 1).

\section{IBD COMBINED WITH CMV INFECTION}

\section{The positive rate of serum anti-CMV IgG in IBD patients is higher than that in healthy controls}

The epidemiology of CMV infection is associated with socioeconomy. ${ }^{5}$ Positive rate of serum CMV IgG in IBD patients has been reported to be high. For example, Yi et al. ${ }^{6}$ reported that serum CMV IgG positive rate was $73.54 \%$ in UC and $89.19 \%$ in CD patients in Wuhan (Hubei Province, China), while that in the healthy population was only $50.69 \%$. However, CD patients are rarely infected with CMV, comprising of $<5 \%$ of the reported cases. ${ }^{7}$

\section{Screening for CMV infection is recommended for acute severe UC patients with glucocorticoid resistance}

Many studies have noted that the rate of active CMV infec- tion has been increasing in patients with severe UC and/or glucocorticoid resistance. According to the reports abroad, the percentages of CMV colitis in severe UC patients with glucocorticoid resistance ranged $20 \%$ to $40 \%,{ }^{8}$ whereas that in UC patients who received emergency colectomy was 27\%. ${ }^{9}$ Data from China showed that active CMV infection affected $46.2 \%$ of the severe UC patients receiving surgery ${ }^{10}$ and $36.7 \%$ of the refractory UC patients. ${ }^{11}$

\section{Positive anti-CMV IgM and/or CMV pp65 antigenemia ( $\geq 1 \mathrm{CMV}$-positive cells out of every 150,000 white blood cells) and/or positive plasma CMV DNA in real-time quantitative PCR (qPCR) suggests active CMV infection}

Several detection techniques are available for active CMV infections, each of them has its advantages and disadvantages. A combination application of various methods can increase the detection rate. (1) CMV-specific serum antibodies: including IgM and IgG. Serum IgM is mainly present after infected for 2 to 4 weeks; therefore, its early diagnostic value is limited. ${ }^{12,13}$ (2) CMV pp65 antigenemia assay: its sensitivity and specificity are $60 \%$ to $100 \%$ and $83 \%$ to $100 \%$, respectively. ${ }^{14}$ The disadvantage of the assay is that it cannot distinguish latent infection from active infection, and the result is affected by the reduced peripheral neutrophil count. (3) Culture of viruses: the specificity of the culture is high $(89 \%-100 \%)$, but with a relatively low sensitivity $(45 \%-78 \%),{ }^{13}$ and it is not widely applied in clinical setting. (4) qPCR detection of plasma and fecal CMV DNA: the sensitivity and specificity of the test to diagnose active infection using qPCR for plasma CMV DNA range $65 \%$ to $100 \%$ and $40 \%$ to $92 \%$, respectively. ${ }^{14,15}$ The sensitivity of CMV DNA in stool samples using qPCR is high. ${ }^{16}$

\section{Gold standard for diagnosing CMV colitis is positive histopathology by H\&E stain combined with positive immunohistochemistry (IHC) and/or positive qPCR for CMV DNA in colonic mucosal tissues}

(1) The sensitivity of HE stain is low (range, 10\%-87\%); therefore, the early diagnostic value of the test is limited. However, its specificity reaches $92 \%$ to $100 \% .{ }^{17-19}$ When giant cells, intranuclear inclusion bodies, perinuclear halo, and owl's eye-like changes are observed (Fig. 1), CMV colitis can be diagnosed. ${ }^{20,21}$ (2) IHC stain of colonic mucosal tissues has a high sensitivity $(78 \%-93 \%)^{22}$ and is the gold standard for the diagnosis of CMV colitis. (3) Detection of CMV DNA 
Table 1. Summary of the Recommendations on Opportunistic Infections in IBD

\section{IBD combined with cytomegalovirus (CMV) infection}

1. The positive rate of serum anti-CMV IgG in IBD patients is higher than that in healthy controls.

2. Screening for CMV infection is recommended for acute severe UC patients with glucocorticoid resistance.

3. Positive anti-CMV IgM and/or CMV pp65 antigenemia ( $\geq 1$ CMV-positive cells out of every 150,000 white blood cells) and/or positive plasma CMV DNA in real-time quantitative PCR (qPCR) suggests active CMV infection.

4. Gold standard for diagnosing CMV colitis is positive histopathology by HEtE stain combined with positive immunohistochemistry and/or positive qPCR for CMV DNA in colonic mucosal tissues.

5. Antiviral therapies should be considered in cases with peripheral CMV DNA $>1,200$ copies/mL by qPCR.

6. Typical colonoscopic features including mucosal defect, punched-out ulcer, longitudinal ulcer, cobblestone-like changes and irregular ulcer suggest CMV colitis, routine biopsy and differential diagnosis should be performed.

7. Antiviral therapy should be initiated in time in severe steroid-resistant colitis patients with CMV colitis. Discontinuation and dose reduction of immunosuppressive agents should be considered based on the evaluation of pros and cons.

8. A 3- to 6-week course of antiviral therapy for patients with IBD combined with CMV colitis is recommended.

\section{IBD combined with Epstein-Barr virus (EBV) infection}

9. IBD patients who present active EBV infection during the administration of immunosuppressive agents are recommended to weigh the pros and cons for discontinuing immunosuppressive agents.

10. Hematologists should be consulted for the diagnosis and treatment of EBV-related lymphoproliferative diseases.

\section{IBD combined with viral hepatitis}

11. All IBD patients should be tested for HBsAg, anti-HBs and anti-HBc. Those with positive HBsAg and anti-HBc should be further tested for HBeAg, anti-HBe and HBV DNA.

12. Prophylactic antiviral therapy with nucleos(t)ide analogues is recommended prior to the use of immunomodulators in $\mathrm{HBsAg-positive}$ IBD patients regardless of HBV DNA levels. Antiviral therapy should be started 1-2 weeks before the treatment with glucocorticoids and immunosuppressive agents and should last for at least 12 months after the cessation of immunosuppressive agents.

13. HCV is not an absolute contraindication to immunosuppressive therapy but should be closely monitored due to a high risk of HCV reactivation.

14. Whether interferon, the commonly used anti-HCV medication, will aggravate IBD remains unclear. The risk of IBD aggravation by anti-HCV therapy and the interaction among drugs should be fully considered. Direct-acting antiviral agents for anti-HCV therapy are recommended.

\section{IBD combined with bacterial infection}

15. When IBD patients have combined active bacterial infections, immunosuppressive agents should be reduced or even discontinued based on patient conditions, and sensitive antibiotics should be chosen.

16. IBD is an independent risk factor of Clostridium difficile infection.

17. Hand hygiene is important for preventing nosocomial infection of $C$. difficile.

18. In IBD patients receiving glucocorticoids or immunosuppressive agents exhibit disease recurrence or unsatisfactory treatment response, screening for $C$. difficile is recommended.

19. Tests for $C$. difficile detection include the detection of dehydrogenase antigen, toxins A/B by ELISA, bacterial culture, cytotoxicity assay and nucleic acid amplification technology.

20. The treatment for IBD patients combined with $C$. difficile infection may refer to that in non-IBD patients; metronidazole or oral vancomycin is recommended. For patients with severe $C$. difficile infection, vancomycin has a better efficacy than metronidazole and is recommended as the preferred choice.

21. For IBD patients combined with $C$. difficile infection, the pros and cons should be considered for the use of immunosuppressive agents.

in colonic mucosal tissues using qPCR has a sensitivity of $92.0 \%$ to $96.7 \%$ and a specificity of $93.0 \%$ to $98.7 \%$.

\section{Antiviral therapies should be considered in cases with peripheral CMV DNA $>1,200$ copies $/ \mathrm{mL}$ by qPCR}

Yang et al. ${ }^{23}$ applied the receiver operating characteris- 
Table 1. Continued

\section{IBD and Mycobacterium tuberculosis infection}

22. Tumor necrosis factor $\alpha$ (TNF- $\alpha$ ) antagonists can cause reactivation of latent tuberculosis infection (LTBI) or increase the risk of tuberculosis infection; therefore, screening for tuberculosis should be routinely performed prior to the initiation of TNF- $\alpha$ antagonists.

23. Screening of tuberculosis is recommended prior to the use of glucocorticoids, purines or methotrexate.

24. Recommendation for screening active tuberculosis or LTBI: past history of tuberculosis infection or contact, chest X-ray examination, pure protein derivative (PPD) test and/or interferon- $\gamma$ release assays (IGRAs) should be performed. The efficacy of IGRAs in the diagnosis of LTBI is better than the PPD test; therefore, IGRAs should be the first diagnostic modality of choice under certain circumstances.

25. Before LTBI patients receive TNF- $\alpha$ antagonists or glucocorticoids (equivalent to prednisone $\geq 15 \mathrm{mg} /$ day), the treatment with 1 to 2 antituberculosis drugs for 3 weeks is recommended. This anti-tuberculosis regimen should continue for 6 months during the TNF- $\alpha$ antagonists or glucocorticoid therapy.

26. When active tuberculosis is diagnosed, the standardized anti-tuberculosis therapy should be started immediately, and TNF- $\alpha$ antagonists and immunosuppressive agents (such as purines and methotrexate) should be discontinued. The pros and cons of continuous use of glucocorticoids in this situation should be weighed or decided after discussion with specialists.

27. Biological agents can be restored after 2-3 months of standard anti-tuberculosis therapy and tuberculosis-related indicators are improved when required when active TB is diagnosed.

\section{IBD combined with fungal infection}

28. Fungi are resident flora in the human gastrointestinal tract that play important roles in intestinal homeostasis. Their functions in the development of IBD are still not clear; they might be able to become opportunistic pathogens in IBD patients.

29. Once IBD patients have a combined invasive fungal infection, drugs that suppress human immunity should be stopped in principle, and antifungal treatment should be started in a timely manner.

\section{IBD combined with parasitic infection}

30. Specific screening for parasitic infections before the application of immunosuppressive agents is not considered necessary unless the patients are long-time residents of or have travelled to epidemic areas.

\section{Vaccination in IBD patients}

31. Live attenuated vaccines are contraindications to those patients with IBD under the use of immunosuppressants.

32. If IBD patients have a negative HBV serology (both anti-HBs and anti-HBc are negative), medical therapy can be started, and inoculation of HBV vaccine is recommended.

33. IBD patients can be inoculated with the pneumococcus vaccine.
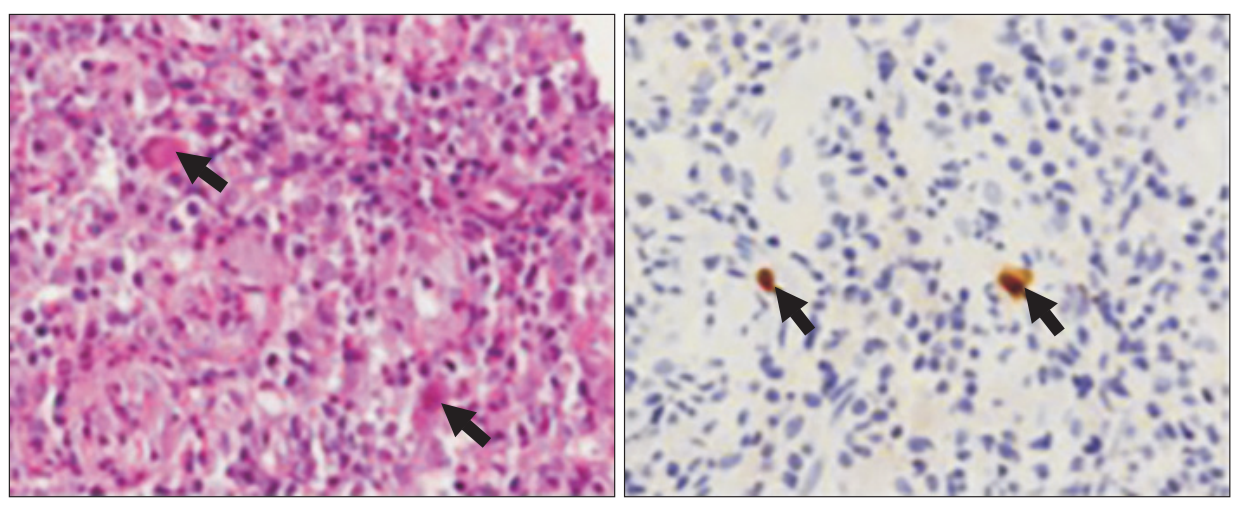

Fig. 1. Histopathology of colonic mucosal tissues (×400). (A) Intranuclear inclusion bodies (arrows, HEtE stain). (B) Immunohistochemistry of positively stained cells (arrows). 
tic (ROC) curve analysis to determine the threshold value of 1,150 copies/mL to detect CMV DNA using qPCR and showed that the sensitivity and specificity for the diagnosis of CMV colitis were $44.4 \%$ and $78.9 \%$, respectively.

\section{Typical colonoscopic features including mucosal defect, punched-out ulcer, longitudinal ulcer, cobblestone-like changes and irregular ulcer suggest CMV Colitis, routine biopsy and differential diagnosis should be performed}

Wide mucosal defect, punched-out ulcer, longitudinal ulcer, cobblestone-like changes and irregular ulcer might be characteristic endoscopic features of CMV colitis. Suzuki et $\mathrm{al}^{24}$ showed that the sensitivity and specificity of longitudinal ulcer in the prediction of CMV colitis reached $100 \%$ and $95 \%$, respectively. Yang et al. ${ }^{23}$ reported that UC patients with CMV colitis can present with punched-out ulcer, irregular ulcer and cobblestone-like changes.

\section{Antiviral therapy should be initiated in time in severe steroid-resistant colitis patients with CMV colitis. Discontinuation and dose reduction of immunosuppressive agents should be considered based on the evaluation of pros and cons}

There is evidence indicating that $\mathrm{CMV}$, as a hidden factor, can aggravate the disease in patients with refractory UC. ${ }^{12,25,26}$ After antiviral therapy, the clinical remission rate reaches $67 \%$ to $100 \%$, and the mortality rate is reduced from $71 \%$ to $14.5 \%-17.6 \%{ }^{8,27}$

The consensus of the European Crohn and Colitis Organization (ECCO) recommends that when severe UC patients with glucocorticoid resistance have combined CMV colitis, antiviral therapy should be administered; the cessation of immunosuppressive agents is also recommended ${ }^{28}$ However, one study suggested that when glucocorticoids or immunosuppressive agents are discontinued, the severity of UC was aggravated. ${ }^{29}$ Therefore, individualized assessment should be performed on the basis of patient's condition.

\section{A 3- to 6-week course of antiviral therapy for patients with IBD combined with CMV colitis is recommended}

The major drugs for treating IBD combined with CMV colitis are ganciclovir and foscarnet sodium. ${ }^{30}$ Ganciclovir is given at a dose of $5 \mathrm{mg} / \mathrm{kg}$ twice daily via intravenous infusion, and the treatment course is usually no less than 3 weeks. Valganciclovir is the prodrug of ganciclovir. Its bioavailability is good by oral administration. After absorption, it is phosphorylated to form ganciclovir triphosphate, which has an efficacy equivalent to that of ganciclovir. The routine dose of the drug is $900 \mathrm{mg}$ twice daily, and it can be administered orally as maintenance therapy. The efficacy of foscarnet sodium is equivalent to that of ganciclovir, and is intravenously administrated at $180 \mathrm{mg} / \mathrm{kg} / \mathrm{day}$. The drug administration is divided into 2 to 3 doses, and the treatment course is usually no less than 3 weeks.

\section{IBD COMBINED WITH EBV INFECTION}

\section{IBD patients who present active EBV infection during the administration of immunosuppressive agents are recommended to weigh the pros and cons for discontinuing immunosuppressive agents}

Several studies have indicated that IBD patients are at a risk of developing lymphoma, especially those who receive mercaptopurine treatment, some might be associated with EBV infection. ${ }^{31-33}$ When IBD patients treated with immunosuppressive agents are suspected to have EBV infection, blood routine examination, peripheral blood smear, liver function test and serological indicator for EBV should be closely monitored. For patients with previously negative EBV serology, the presence of elevated EBV DNA titers might indicate the risk of developing lymphoproliferative diseases. ${ }^{34}$ The primary treatment is to reduce the dose of or discontinue immunosuppressive agents. After immunosuppressive agents are discontinued, EBV-related lymphoproliferative diseases can usually be spontaneously relieved. ${ }^{35}$

\section{Hematologists should be consulted for the diagnosis and treatment of EBV-related lymphoproliferative diseases}

IBD patients who have active EBV infection poorly responded to antiviral therapy (acyclovir and ganciclovir), while antiviral therapy is ineffective when EBV-related lymphoproliferative diseases are present. ${ }^{34}$ Discontinuation of immunosuppressive agents might lead to a spontaneous regression of EBV-related lymphoproliferative diseases. ${ }^{35,36}$ In the absence of spontaneous regression or progression after the withdrawal of immunosuppressive agents, rituximab monoclonal antibody can be considered for CD20-positive B cell lymphoma. In addition, patients with EBV infection 
should be highly alerted to the development of macrophage activation syndrome (MAS) and hemophagocytic lymphohistiocytosis (HLH). Once EBV infection combined with MAS/HLH or EBV-related lymphoproliferative diseases occurs, close collaboration and correspondence with hematologists are recommended to establish reasonable diagnostic and therapeutic strategies.

\section{IBD COMBINED WITH VIRAL HEPATITIS}

\section{All IBD patients should be tested for HBsAg, anti-} HBs and anti-HBc. Those with positive HBsAg and anti-HBc should be further tested for HBeAg, antiHBe and HBV DNA

Foreign studies have reported 6 cases of IBD patients exhibiting HBV reactivation during the administration of glucocorticoids and/or thiopurines, 5 of whom developed liver failure. ${ }^{37-39} \mathrm{HBV}$ reactivation in IBD patients caused by infliximab (IFX) has also been reported. ${ }^{40}$ A clinical survey of IFX application in China showed that 3 out of $4 \mathrm{HBsAg}$-positive patients presented elevated ALT while using IFX. ${ }^{41}$ Therefore, HBV screening is recommended to be performed when IBD is initially diagnosed rather than after immunosuppressive agent therapy is initiated. ${ }^{42-45}$ Since occult infection carries a risk of HBV reactivation, further HBV DNA screening has been recommended for patients with negative HBsAg and positive anti-HBc. ${ }^{38}$

\section{Prophylactic antiviral therapy with nucleos(t)ide analogues is recommended prior to the use of immunomodulators in HBsAg-positive IBD patients regardless of HBV DNA levels.}

Antiviral therapy should be started 1-2 weeks before the treatment with glucocorticoids and immunosuppressive agents and should last for at least 12 months after the cessation of immunosuppressive agents

HBV reactivation rate has been reported to be $16 \%$ to $36 \%$ in HBsAg-positive IBD patients. The reactivation risk is associated with long-term ( $>3$ months) combined $(\geq 2)$ application of immunosuppressive agents without receiving prophylactic antiviral therapy. ${ }^{45}$ Lamivudine is the most commonly used prophylactic antiviral drug for IBD; however, its 1-year and 5-year drug resistance rates are up to $30 \%$ and $70 \%$, respectively. ${ }^{46}$ Long-term anti-tumor necrosis factor (anti-TNF) therapy can further increase the drug re- sistance rate. ${ }^{47,48}$ Therefore, lamivudine is currently recommended for short-term treatment only. For IBD patients, the influence of antiviral therapy on the immunosuppressive treatment should be avoided as much as possible. Therefore, tenofovir and entecavir, which have low drug resistance rates and strong antiviral effects, are recommended. ${ }^{28,49,50}$ The risk of developing liver cirrhosis and hepatocellular carcinoma (HCC) in chronic hepatitis B with HBV DNA $>2,000 \mathrm{IU} / \mathrm{mL}$ is significantly increased; therefore, patients with HBV DNA $>2,000 \mathrm{IU} / \mathrm{mL}$ should continue antiviral therapy. The endpoint of treatment is the same as that of the general hepatitis B population. ${ }^{28,51,52}$

\section{HCV is not an absolute contraindication to immunosuppressive therapy but should be closely monitored due to a high risk of $\mathrm{HCV}$ reactivation}

In 2014, Huang et al. ${ }^{53}$ reported that HCV infection rate in IBD patients was not statistically different from that in the general population. Among 714 IBD patients, the HCV infection rate was $0.42 \%$, while that in the non-IBD patients was $0.36 \%(P=0.80)$. This result was consistent with another Italian study. ${ }^{54}$

The use of glucocorticoids and immunosuppressive agents in IBD patients may affect the disease course of hepatitis C. Loras et al. ${ }^{38}$ reavealed that the use of glucocorticoids might result in massive replication of $\mathrm{HCV}$ and liver damage. Brunasso et al. ${ }^{55}$ analyzed 37 relevant studies in $153 \mathrm{HCV}$ infected patients who received IFX for rheumatoid arthritis and showed that only one exhibited definite hepatic aggravation. Current data has shown the acceptable safety of antiTNF therapy in HCV-infected IBD patients. ${ }^{45,55}$

\section{Whether interferon (IFN), the commonly used anti-HCV medication, will aggravate IBD remains unclear. The risk of IBD aggravation by anti-HCV therapy and the interaction among drugs should be fully considered. Direct-acting antiviral agents (DAAs) for anti-HCV therapy are recommended}

Currently, the major anti-HCV regimen in China is the PR regimen, consisting of pegylated interferon $\alpha$ (PEG IFN- $\alpha$ ) combined with ribavirin. ${ }^{56}$ This regimen is applicable to all HCV genotypes when there are no contraindications. A newly developed mediation, anti-HCV DAAs, is already on the Western markets, but this type of drugs is still under clinical trial in China. Before they receive antiviral therapy for HCV infection, the risk of IBD aggravation by antiviral therapy and 
the possible interactions among drugs should be fully considered. $^{45}$

\section{IBD COMBINED WITH BACTERIAL INFECTION}

\section{When IBD patients have combined active bacterial infections, immunosuppressive agents should be reduced or even discontinued based on patient conditions, and sensitive antibiotics should be chosen}

Both immunosuppressive and biological agents can cause immune decline, when IBD patients have combined active bacterial infections, immunosuppressive agents should be temporarily suspended, and sensitive antibiotics should be applied. $^{28}$

\section{IBD is an independent risk factor of Clostridium difficile infection}

C. difficile is a type of Gram-positive, spore-forming, anaerobic bacillus that is a common opportunistic pathogen of nosocomial infections. $C$. difficile can induce diarrhea, pseudomembranous colitis and severe sepsis. The risk factors of C. difficile infection include antibiotic exposure, low immunity, long-term hospitalization and advanced age. ${ }^{57,58}$ Studies from the Western world have shown that IBD, especially $\mathrm{UC}$, is an independent risk factor of $C$. difficile infection. ${ }^{28} \mathrm{~A}$ single-center, retrospective study suggested that $C$. difficile infection rate is significantly increased in IBD patients; in particular, those with active disease and colonic involvement are prone to be infected with $C$. difficile. ${ }^{59}$

\section{Hand hygiene is important for preventing nosocomial infection of $C$. difficile}

There are many transmission vectors of $C$. difficile, among which transmission by hand is an important route. ${ }^{60}$ Protection through the use of gloves or hand hygiene is important for preventing nosocomial infection. Prevention with drugs is currently not recommended for $C$. difficile infection. Guo et al. ${ }^{61}$ compared the effects of 5 different hand hygiene methods for removing $C$. difficile on hands, showing that general liquid soap had the best effect on $C$. difficile, followed by antibacterial liquid soap, quaternary ammonium salt disinfecting wipes, running water, and the 6-step hand-hygiene technique using an alcohol-based rapid hand-disinfection solution. Isolation of the patient is recommended to prevent the transmission of infection within the hospital if $C$. difficile infection is confirmed or suspected. ${ }^{62}$

\section{In IBD patients receiving glucocorticoids or} immunosuppressive agents exhibit disease recurrence or unsatisfactory treatment response, screening for $\boldsymbol{C}$. difficile is recommended

IBD patients with long-term use of glucocorticoids or immunosuppressive agents are at a significantly increased risk of $C$. difficile infection that may aggravate the severity of the disease. A large-scale study based on the general population showed that, regardless of doses and treatment courses, the risk of $C$. difficile infection in IBD patients treated with glucocorticoids was increased by 3.4 -fold compared with those receiving immunosuppressive and biological agents. ${ }^{63}$ Longterm use of immunosuppressive agents also increases the risk of $C$. difficile infection in IBD patients, ${ }^{64}$ while long-term application of biological agents does not. ${ }^{63}$

\section{Tests for $C$. difficile detection include the detection of dehydrogenase antigen, toxins A/B by ELISA, bacterial culture, cytotoxicity assay and nucleic acid amplification technology (NAT)}

The detection of $C$. difficile detection includes the following 3 methods: (1) detection of toxins $\mathrm{A} / \mathrm{B}$ of $C$. difficile in stool samples or via the cytotoxicity neutralization assay (CCNA); (2) detection of the bacterium, such as the detection or culture of glutamate dehydrogenase (GDH); (3) detection of toxin genes using NAT ${ }^{65} \mathrm{Xu}$ et al.$^{66}$ compared the 3 detection methods (C. difficile stool culture, nucleic acid PCR, and detection of toxin A/B using ELISA) and demonstrated that the sensitivity of nucleic acid PCR and ELISA toxin detection was not significantly different from that of anaerobic culture.

There are many methods for the diagnosis of $C$. difficile infection, among which detecting toxin $B$ of the bacterium using CCNA is the gold standard. Combination use of NAT and ELISA for combined detection is generally recommended. Endoscopic examination is generally not used as an essential detection method for $C$. difficile infection. 
20. The treatment for IBD patients combined with C. difficile infection may refer to that in nonIBD patients; metronidazole or oral vancomycin is recommended. For patients with severe $C$. difficile infection, vancomycin has a better efficacy than metronidazole and is recommended as the preferred choice

Metronidazole is the preferred choice of treatment for $C$. difficile infection, including recurrent infections. ${ }^{67}$ Metronidazole is generally given orally at a dose of 200 to $250 \mathrm{mg} 4$ times/day or 400 to $500 \mathrm{mg}$ thrice daily fora total treatment course of 10-14 days. Vancomycin can be used for treating recurrent or metronidazole-ineffective $C$. difficile infection. ${ }^{68}$ A meta-analysis has shown that the efficacy of treating mildto-moderate $C$. difficile using vancomycin or metronidazole was not significantly different from that of using other antibiotics (such as rifaximin). ${ }^{69-71}$ However, for patients with severe $C$. difficile infection or have aggravated symptoms after treated with metronidazole, vancomycin is recommended to be given as soon as possible. ${ }^{72}$ For acute $C$. difficile infection, oral administration of vancomycin at the dose of $125 \mathrm{mg}$ every 6 hours is recommended. ${ }^{72}$ To prevent the recurrence of $C$. difficile infection, gradually reducing the dose of vancomycin or using intermittent medication is recommended. The specific usage is the oral administration of 125 to 500 mg every 3 days for 2 to 3 weeks. ${ }^{68}$ Other antibiotics such as nitazoxanide and rifaximin are mainly used for recurrent $C$. difficile infection. ${ }^{73}$ Intravenous administration of tigecycline is effective for severe, complicated, and recurrent $C$. difficile infection. $^{74}$

\section{For IBD patients combined with $C$. difficile} infection, the pros and cons should be considered for the use of immunosuppressive agents

The application of thiopurines in IBD patients can increase the risk of $C$. difficile infection. ${ }^{75}$ If antibiotics only are used and without the administration of immunosuppressive agents, the above-mentioned risk is not increased. If immunosuppressive agents are used in combination, such risk might be further increased. ${ }^{76}$ Therefore, whether IBD patients combined with $C$. difficile infection should continue using immunosuppressive agents or not would be considered based on the evaluation of the pros and cons of treatment efficacy and the adverse consequences caused by $C$. difficile infection.

\section{IBD AND M. tuberculosis INFECTION}

\section{TNF- $\alpha$ antagonists can cause reactivation of latent tuberculosis infection (LTBI) or increase the risk of tuberculosis infection; ${ }^{77}$ therefore, screening for tuberculosis should be routinely performed prior to the initiation of TNF- $\alpha$ antagonists}

Recent studies have shown that some IBD patients (1.65\%) still develop tuberculosis even after LTBI screening before the use of TNF- $\alpha$ antagonists; ${ }^{78}$ these patients are prone to develop extra-pulmonary tuberculosis that presents as fever, elevated CRP and low positive rate of pathogen detection. ${ }^{79}$ IBD patients with LTBI should be given anti-tuberculosis therapy for at least 3 weeks before the administration of TNF- $\alpha$ antagonists. And patients should avoid biological agent treatment within 3 months after receiving $M$. tuberculosis vaccination. ${ }^{28,80,81}$ During the TNF- $\alpha$ antagonist therapy, tuberculosis infection should be monitored by evaluating patient's clinical presentations and chest X-ray examination every 8 to 16 weeks. ${ }^{82,83}$

\section{Screening of tuberculosis is recommended prior to} the use of glucocorticoids, purines or methotrexate

It has been shown that the application of glucocorticoid equivalent to prednisone $\geq 15 \mathrm{mg}$ /day for more than one month can increase the risk of LTBI. ${ }^{84-86}$ Using purines alone can also increase the risk of LTBI reactivation. ${ }^{87,88}$ The combination of purines with glucocorticoids and/or TNF- $\alpha$ antagonists is more likely to cause LTBI reactivation than purines monotherapy.

\section{Recommendation for screening active tuberculosis or LTBI: past history of tuberculosis infection or contact, chest $\mathrm{X}$-ray examination, pure protein derivative (PPD) test and/or interferon- $\gamma$ release assays (IGRAs) should be performed. The efficacy of IGRAs in the diagnosis of LTBI is better than the PPD test; therefore, IGRAs should be the first diagnostic modality of choice under certain circumstances}

Patients' past history of tuberculosis infection or contact, the presence of tuberculosis poisoning symptoms and chest $\mathrm{X}$-ray results should all be considered in screening active tuberculosis infection. ${ }^{28,89,90}$ Current screening methods for LTBI include the tuberculin skin test (TST) and IGRAs 
(quantiFERON-TB Gold and T-SPOT.TB). Neither TST nor IGRAs can distinguish between LTBI and active tuberculosis. Many studies have shown that IGRAs are better than TST in cases received BCG inoculation, those with a contact history of active tuberculosis, high-risk medical workers and children infected with tuberculosis. ${ }^{91-97}$ Considering China as a country with heavy tuberculosis burden, the Chinese Society for Tuberculosis, Chinese Medical Association recommends both PPD and IGRAs to be adopted for screening LTBI in China. Patients with a positive PPD test can be further evaluated by IGRAs for further confirmation. Patients with autoimmune diseases or organ transplantation should receive IGRAs alone or in combination with PPD tests for screening LTBI before receiving glucocorticoids or TNF- $\alpha$ antagonists. $^{98}$

25. Before LTBI patients receive TNF- $\alpha$ antagonists or glucocorticoids (equivalent to prednisone $\geq 15 \mathrm{mg}$ / day), the treatment with 1 to 2 anti-tuberculosis drugs for 3 weeks is recommended. This antituberculosis regimen should continue for 6 months during the TNF- $\alpha$ antagonists or glucocorticoid therapy

For IBD patients having LTBI who are being treated with TNF- $\alpha$ antagonists or glucocorticoids, the following prophylactic anti-tuberculosis strategies are recommended: isoniazid $0.3 \mathrm{~g} /$ day and rifampin $0.45 \mathrm{~g} /$ day for 6 months, or isoniazid $0.9 \mathrm{~g}$ /week and rifapentine $0.9 \mathrm{~g} /$ week for 3 to 6 months. Whether prophylactic anti-tuberculosis treatment is required for IBD patients with previous tuberculosis should be individualized based on patients' past treatments and discussions with specialists. ${ }^{99}$

\section{When active tuberculosis is diagnosed, the} standardized anti-tuberculosis therapy should be started immediately, and TNF- $\alpha$ antagonists and immunosuppressive agents (such as purines and methotrexate) should be discontinued. The pros and cons of continuous use of glucocorticoids in this situation should be weighed or decided after discussion with specialists

Currently, there has been no recommendation for a standard regimen and treatment course of anti-tuberculosis therapy targeting immunosuppressed hosts. Patients are recommended to be transferred or referred to tuberculosisspecific hospitals or to receive medications under the guid- ance of tuberculosis specialists. Patients with treatmentnaive tuberculosis can be given the 2HRZE/4HR regimen $(\mathrm{H}$, isoniazid; $\mathrm{R}$, rifampicin; $\mathrm{Z}$, pyrazinamide; $\mathrm{E}$, ethambutol) for 6 months according to the recommendations of the World Health Organization (WHO) and the Chinese guidelines for the prevention and treatment of pulmonary tuberculosis. Patients with recurrent pulmonary tuberculosis are provided with the 3HRZES/6HRE regimen (S, streptomycin) for 9 months. Patients with tuberculous pleuritis are given the 2HRZE/10HRE regimen for 12 months. Because IBD patients infected with active tuberculosis are mainly exhibiting opportunistic infection in an immunosuppressive host, the 2HRZE/10HRE anti-tuberculosis regimen for 12 months is recommended.

\section{Biological agents can be restored after 2-3} months of standard anti-tuberculosis therapy and tuberculosis-related indicators are improved when required when active $T B$ is diagnosed

According to the London consensus of the World Congress of Gastroenterology on biological therapy for $\mathrm{IBD}^{80}$ and the ECCO consensus on IBD opportunistic infection, ${ }^{28}$ the restoration of TNF- $\alpha$ antagonists is recommended to be considered after 2 to 3 months of standard anti-tuberculosis therapy and when the patient's tuberculosis-related indicators are improved. Currently, there is no evidence showing the appropriate timing to restore immunosuppressive agents in IBD patients who are infected with tuberculosis; the patient's overall condition should be considered, and the consensus on biological agents may be used as a reference.

\section{IBD COMBINED WITH FUNGAL INFECTION}

\section{Fungi are resident flora in the human gastrointestinal tract that play important roles in intestinal homeostasis. Their functions in the development of IBD are still not clear; they might be able to become opportunistic pathogens in IBD patients}

When human immunity decreases (e.g., after treated with immunosuppressive agents and glucocorticoids and in the presence of gene mutations in effector molecules including Dectin-1 and CARD9) and/or the fungal load increases (such as with the use of large amounts of antibiotics that causes intestinal flora disturbance, thus resulted in excessive fungal growth), normal fungal flora in the intestine and other locations may turn into pathogens, inducing fungal infec- 
tious diseases and even lethal invasive fungal infection. ${ }^{100-104}$

\section{Once IBD patients have a combined invasive fungal infection, drugs that suppress human immunity should be stopped in principle, and anti-fungal treatment should be started in a timely manner}

Based on the different locations of infections and disease severity, treatment strategies for fungal infection also differ. Focal superficial fungal infections usually require local application of anti-fungal drugs, whereas disseminated fungal infections usually require the intravenous administration of anti-fungal drugs. Based on such situations, surgical debridement and immunotherapy may also be required. ${ }^{105,106}$

Once invasive fungal infection is confirmed, drugs that suppress human immune functions should be stopped in principle, including glucocorticoids, immunosuppressive and biological agents. If fungal infection is only superficial (such as local skin infection), local anti-fungal drugs can effectively control the disease; whether immunosuppressive agents should be stopped remains controversial and requires a careful assessment of IBD severity and the pros and cons of continuous therapy. However, the risk of continuous application of anti-TNF preparations is high.

\section{IBD COMBINED WITH PARASITIC INFECTION}

\section{Specific screening for parasitic infections before the application of immunosuppressive agents is not considered necessary unless the patients are long- time residents of or have travelled to epidemic areas}

There are relatively fewer studies on IBD combined with parasitic infection. Currently, there is no clear evidence supporting routine screening for parasites. If patients are long-time residents of or have travelled to an epidemic area, screening can be considered conditionally. If patients are suspected to have a combined parasitic infection, immunosuppressive agents can be reduced accordingly. After the infection is controlled and IBD needs to be treated using immunosuppressive agents in a patient, secondary prevention can be performed based on the opinions of infectious disease specialists.

\section{VACCINATION IN IBD PATIENTS}

\section{Live attenuated vaccines are contraindications to those patients with IBD under the use of immunosuppressants}

IBD patients exhibit dysregulated immune responses after treatment with immunosuppressants. Vaccines for preventing against pathogens of opportunistic infection can be considered. ${ }^{107,108}$ If the inoculation of live attenuated vaccines is required during the immunosuppressive agent treatment, glucocorticoids are recommended to be discontinued for 1 month and immunosuppressive agents for more than 3 months. If the inoculation of live attenuated vaccines is required before using immunosuppressive agents, the drugs should be postponed for at least 3 weeks.

\section{If IBD patients have a negative HBV serology (both anti-HBs and anti-HBc are negative), medical therapy can be started, and inoculation of $\mathrm{HBV}$ vaccine is recommended}

For IBD patients with negative HBV serology (both antiHBs and anti-HBc are negative), inoculation of HBV vaccine (recombinant [yeast] hepatitis B vaccine) is recommended to be given at the time of IBD diagnosis. ${ }^{51,109}$

The HBV vaccine inoculation procedure involves several steps. A double-dose inoculation and/or re-inoculation procedure may be preferential, that is, 3 doses of $40 \mu \mathrm{g}$ recombinant hepatitis B vaccine are inoculated at 0,1 and 2 months at the first vaccination, and anti-HBs needs to be re-examined within 1 to 3 months after the last shot. Patients with anti-HBs $<100 \mathrm{IU} / \mathrm{L}$ must receive the second round of vaccination. The total response rate after 2 inoculations is $57 \%$ to $79 \% .^{110,111}$ Moreover, other guidelines also recommend receiving another 3 doses if the first inoculation fails. ${ }^{112,113}$

Screening anti-HBs levels every 6 to 12 months has been recommended to determine the immunosuppressive status, and those with anti-HBs $<10 \mathrm{IU} / \mathrm{L}$ should receive one booster shot. However, there is no relevant recommendation for IBD patients. ${ }^{112,114}$

Regarding the inoculation time, the short course $(0,1$ and 2 months) allows immunosuppressive patients to obtain immune protection as soon as possible, whereas the long course (0, 1 and 6 months) yields higher antibody titers but increases the risk of $\mathrm{HBV}$ infection in patients with delayed immune responses. ${ }^{112}$ In addition, a four-shot inoculation ( 0 , 1, 2 and 6 months) may increase the immune response, ${ }^{112}$ al- 
though this strategy requires further studies in IBD patients.

Regarding antibody titers for IBD patients, anti-HBs level $>10 \mathrm{IU} / \mathrm{L}$ is considered to confer effective immune protection. ${ }^{111}$ However, for high-risk populations, such as patients with immunosuppressive status and chronic diseases (including IBD), anti-HBs $>100 \mathrm{IU} / \mathrm{L}$ is considered to confer effective serological protection. ${ }^{115,116}$

\section{IBD patients can be inoculated with the pneumococcus vaccine}

PPV23 is a 23-valent polysaccharide vaccine targeting Streptococcus pneumonia that contains up to $98 \%$ of S. pneumoniae serotypes that cause pneumonia. PPV23 stimulates excellent immune functions in clinical trials in IBD patients. ${ }^{117}$ ECCO consensus recommends inoculating the PPV23 pneumococcus vaccine 2 weeks before the initiation of treatment in IBD patients to prevent infection with $S$. pneumonia. $^{28}$

\section{FINANCIAL SUPPORT}

This work was supported by a Health Research \& Special Projects Grant of China (No.201002020).

\section{CONFLICT OF INTEREST}

No potential conflict of interest relevant to this article was reported.

\section{AUTHOR CONTRIBUTION}

Written by (based on the chapter order): Hong Yang, Zhi Hua Ran, Yu Lan Liu, Kai Chun Wu, Xiao Ping Wu, Yao He, Zhan Ju Liu.

Voted by (ordered by pinyin of last names): Min Hu Chen, Liu Fang Cheng, Qian Cao, Yan Chen, Ye Chen, Ning Chen, Xiao Cang Cao, Min Chen, Chang Sheng Deng, Yi Qi Du, Yan Dou, Yi Hong Fan, Zhe Feng, Xiang Gao, Hua Tian Gan, Fang Gu, Wei Han, Ying Han, Nai Zhong Hu, Pin Jin Hu, Yi Qun Hu, Mei Fang Huang, Li Juan Huo, Xue Liang Jiang, Jin Li, Jun Xia Li, Yan Qing Li, Yue Li, Jie Liang, Xin Guang Liu, Hong Lv, Ying Lei Miao, Qiao Mei, Qin Ouyang, Zhi Pang, Jun Shen, Hua Xiu Shi, Feng Tian, Yi Tu, Cheng Dang Wang, Hua Hong Wang, Yu Fang Wang, Xin Ying Wang, Cheng Gong Yu, Wei Yan Yao, Hong Jie Zhang, Hu Zhang, Xiao Lan Zhang, Ya Li Zhang, Jia Ju Zheng, Ping Zheng, Peng Yuan Zheng, Chang
Qing Zheng, Jie Zhong, Feng Zhou, Fa Chao Zhi, Zhen Hua Zhu, Kai Fang Zou.

Reviewer of evidence-based medicine: Si Yan Zhan.

Reviewers of infection specialty: Xiao Qing Liu, Rui Yuan Sheng, Bao Tong Zhou.

\section{REFERENCES}

1. Cheon JH. Understanding the complications of anti-tumor necrosis factor therapy in East Asian patients with inflammatory bowel disease. J Gastroenterol Hepatol 2017;32:769-777.

2. Wu D, Li Y, Wang LY, Li JN, Qian JM. A pilot case control study of opportunistic infections in severe ulcerative colitis. Chin J Pract Intern Med 2016;36:482-484.

3. Lu H, Liu AL, Li J, Wu D, Zhao YX, Qian JM. Clinical characteristics of ulcerative colitis complicated with opportunistic infections in elderly patients. Zhongguo Yi Xue Ke Xue Yuan Xue Bao 2016;38:288-293.

4. Qian JM, Yang H. Understanding and progress of opportunistic infections in inflammatory bowel disease. Chin J Gastroenterol Hepatol 2016;25:1081-1083.

5. Guo LL, Xu HM. Progress in epidemiological research of human cytomegalovirus infection. Int J Lab Med 2010;31:11311133.

6. Yi F, Zhao J, Luckheeram RV, et al. The prevalence and risk factors of cytomegalovirus infection in inflammatory bowel disease in Wuhan, Central China. Virol J 2013;10:43. doi: 10.1186/1743-422X-10-43.

7. Takahashi Y, Tange T. Prevalence of cytomegalovirus infection in inflammatory bowel disease patients. Dis Colon Rectum 2004;47:722-726.

8. Eyre-Brook IA, Dundas S. Incidence and clinical significance of colonic cytomegalovirus infection in idiopathic inflammatory bowel disease requiring colectomy. Gut 1986;27:14191425.

9. Cooper HS, Raffensperger EC, Jonas L, Fitts WT Jr. Cytomegalovirus inclusions in patients with ulcerative colitis and toxic dilation requiring colonic resection. Gastroenterology 1977; 72:1253-1256.

10. Li J, Lyu H, Yang H, et al. Preoperative corticosteroid usage and hypoalbuminemia increase occurrence of short-term postoperative complications in Chinese patients with ulcerative colitis. Chin Med J (Engl) 2016;129:435-441.

11. Li TT, Lv ZS, Wang BM, Zhang J. Relationship between refractory ulcerative colitis and cytomegalovirus infection. World Chin J Digestol 2010;18:1174-1177. 
12. Domènech E, Vega R, Ojanguren I, et al. Cytomegalovirus infection in ulcerative colitis: a prospective, comparative study on prevalence and diagnostic strategy. Inflamm Bowel Dis 2008;14:1373-1379.

13. Nakase H, Matsumura K, Yoshino T, Chiba T. Systematic review: cytomegalovirus infection in inflammatory bowel disease. J Gastroenterol 2008;43:735-740.

14. Boivin G, Handfield J, Toma E, et al. Evaluation of the AMPLICOR cytomegalovirus test with specimens from human immunodeficiency virus-infected subjects. J Clin Microbiol 1998;36:2509-2513.

15. Lazzarotto T, Dal Monte P, Landini MP. Recent advances in the diagnosis of cytomegalovirus infection. Ann Biol Clin (Paris) 1996;54:259-265.

16. Herfarth HH, Long MD, Rubinas TC, Sandridge M, Miller MB. Evaluation of a non-invasive method to detect cytomegalovirus (CMV)-DNA in stool samples of patients with inflammatory bowel disease (IBD): a pilot study. Dig Dis Sci 2010;55:10531058.

17. Wu GD, Shintaku IP, Chien K, Geller SA. A comparison of routine light microscopy, immunohistochemistry, and in situ hybridization for the detection of cytomegalovirus in gastrointestinal biopsies. Am J Gastroenterol 1989;84:1517-1520.

18. Dieterich DT, Rahmin M. Cytomegalovirus colitis in AIDS: presentation in 44 patients and a review of the literature. J Acquir Immune Defic Syndr 1991;4 Suppl 1:S29-S35.

19. Cotte L, Drouet E, Bissuel F, Denoyel GA, Trepo C. Diagnostic value of amplification of human cytomegalovirus DNA from gastrointestinal biopsies from human immunodeficiency virus-infected patients. J Clin Microbiol 1993;31:2066-2069.

20. Beaugerie L, Cywiner-Golenzer C, Monfort L, et al. Definition and diagnosis of cytomegalovirus colitis in patients infected by human immunodeficiency virus. J Acquir Immune Defic Syndr Hum Retrovirol 1997;14:423-429.

21. Franzin G, Muolo A, Griminelli T. Cytomegalovirus inclusions in the gastroduodenal mucosa of patients after renal transplantation. Gut 1981;22:698-701.

22. Kandiel A, Lashner B. Cytomegalovirus colitis complicating inflammatory bowel disease. Am J Gastroenterol 2006;101:28572865.

23. Yang H, Zhou W, Lv H, et al. The association between CMV viremia or endoscopic features and histopathological characteristics of CMV colitis in patients with underlying ulcerative colitis. Inflamm Bowel Dis 2017;23:814-821.

24. Suzuki H, Kato J, Kuriyama M, Hiraoka S, Kuwaki K, Yamamoto K. Specific endoscopic features of ulcerative colitis complicated by cytomegalovirus infection. World J Gastroenterol 2010; 16:1245-1251.
25. Cottone M, Pietrosi G, Martorana G, et al. Prevalence of cytomegalovirus infection in severe refractory ulcerative and Crohn's colitis. Am J Gastroenterol 2001;96:773-775.

26. Nakase H, Honzawa Y, Toyonaga T, et al. Diagnosis and treatment of ulcerative colitis with cytomegalovirus infection: importance of controlling mucosal inflammation to prevent cytomegalovirus reactivation. Intest Res 2014;12:5-11.

27. Yoshino T, Nakase H, Ueno S, et al. Usefulness of quantitative real-time PCR assay for early detection of cytomegalovirus infection in patients with ulcerative colitis refractory to immunosuppressive therapies. Inflamm Bowel Dis 2007;13:15161521.

28. Rahier JF, Magro F, Abreu C, et al. Second European evidencebased consensus on the prevention, diagnosis and management of opportunistic infections in inflammatory bowel disease. J Crohns Colitis 2014;8:443-468.

29. Hommes DW, Sterringa G, van Deventer SJ, Tytgat GN, Weel J. The pathogenicity of cytomegalovirus in inflammatory bowel disease: a systematic review and evidence-based recommendations for future research. Inflamm Bowel Dis 2004;10:245250.

30. Kim YS, Kim YH, Kim JS, et al. The prevalence and efficacy of ganciclovir on steroid-refractory ulcerative colitis with cytomegalovirus infection: a prospective multicenter study. J Clin Gastroenterol 2012;46:51-56.

31. Beaugerie L, Brousse N, Bouvier AM, et al. Lymphoproliferative disorders in patients receiving thiopurines for inflammatory bowel disease: a prospective observational cohort study. Lancet 2009;374:1617-1625.

32. Kandiel A, Fraser AG, Korelitz BI, Brensinger C, Lewis JD. Increased risk of lymphoma among inflammatory bowel disease patients treated with azathioprine and 6-mercaptopurine. Gut 2005;54:1121-1125.

33. Smith MA, Irving PM, Marinaki AM, Sanderson JD. Review article: malignancy on thiopurine treatment with special reference to inflammatory bowel disease. Aliment Pharmacol Ther 2010;32:119-130.

34. Lee TC, Savoldo B, Rooney CM, et al. Quantitative EBV viral loads and immunosuppression alterations can decrease PTLD incidence in pediatric liver transplant recipients. Am J Transplant 2005;5:2222-2228.

35. Reddy N, Rezvani K, Barrett AJ, Savani BN. Strategies to prevent EBV reactivation and posttransplant lymphoproliferative disorders (PTLD) after allogeneic stem cell transplantation in high-risk patients. Biol Blood Marrow Transplant 2011;17:591597. 
36. Keever-Taylor CA, Behn B, Konings S, Orentas R, Davies B, Margolis D. Suppression of EBV release from irradiated B lymphoblastoid cell-lines: superior activity of ganciclovir compared with acyclovir. Cytotherapy 2003;5:323-335.

37. Sacco R, Bertini M, Bresci G, Romano A, Altomare E, Capria A. Entecavir for hepatitis B virus flare treatment in patients with Crohn's disease. Hepatogastroenterology 2010;57:242-245.

38. Loras C, Gisbert JP, Mínguez M, et al. Liver dysfunction related to hepatitis B and C in patients with inflammatory bowel disease treated with immunosuppressive therapy. Gut 2010;59: 1340-1346.

39. Zeitz J, Mullhaupt B, Fruehauf H, Rogler G, Vavricka SR. Hepatic failure due to hepatitis B reactivation in a patient with ulcerative colitis treated with prednisone. Hepatology 2009;50:653654.

40. Shu HJ, Li J, Yang H, Qian JM. Risk of hepatitis B virus reactivation in patients with inflammatory bowel disease combined with positive hepatitis B surface antigen after immunosuppressive therapies. Chin J Gastroenterol Hepatol 2016;25:11271131.

41. Chinese Society of Digestive Diseases; IBD Collaboration Group. Investigation on the safety of infliximab against Crohn's disease. Chinese Society of Digestive Diseases. Proceedings of the 9th Congress of Gastroenterology China symposium. Guangdong: Guangdong Medical Association, 2009.

42. Melmed GY, Ippoliti AF, Papadakis KA, et al. Patients with inflammatory bowel disease are at risk for vaccine-preventable illnesses. Am J Gastroenterol 2006;101:1834-1840.

43. Hou JK, Velayos F, Terrault N, Mahadevan U. Viral hepatitis and inflammatory bowel disease. Inflamm Bowel Dis 2010;16:925932.

44. Melmed GY. Vaccination strategies for patients with inflammatory bowel disease on immunomodulators and biologics. Inflamm Bowel Dis 2009;15:1410-1416.

45. Liu XQ, Qin L. Treatment strategy of inflammatory bowel disease associated with viral hepatitis. Chin J Gastroenterol Hepatol 2016;25:1091-1093.

46. Ayoub WS, Keeffe EB. Review article: current antiviral therapy of chronic hepatitis B. Aliment Pharmacol Ther 2011;34:11451158 .

47. Esteve M, Loras C, González-Huix F. Lamivudine resistance and exacerbation of hepatitis B in infliximab-treated Crohn's disease patient. Inflamm Bowel Dis 2007;13:1450-1451.

48. Esteve M, Saro C, González-Huix F, Suarez F, Forné M, Viver JM. Chronic hepatitis B reactivation following infliximab therapy in Crohn's disease patients: need for primary prophylaxis. Gut 2004;53:1363-1365.
49. Coban S, Kekilli M, Köklü S. Approach and management of patients with chronic hepatitis $\mathrm{B}$ and $\mathrm{C}$ during the course of inflammatory bowel disease. Inflamm Bowel Dis 2014;20:21422150.

50. López-Serrano P, Pérez-Calle JL, Sánchez-Tembleque MD. Hepatitis B and inflammatory bowel disease: role of antiviral prophylaxis. World J Gastroenterol 2013;19:1342-1348.

51. Gisbert JP, Chaparro M, Esteve M. Review article: prevention and management of hepatitis $\mathrm{B}$ and $\mathrm{C}$ infection in patients with inflammatory bowel disease. Aliment Pharmacol Ther 2011;33:619-633.

52. European Association for the Study of the Liver. EASL clinical practice guidelines: management of chronic hepatitis B virus infection. J Hepatol 2012;57:167-185.

53. Huang ML, Xu XT, Shen J, Qiao YQ, Dai ZH, Ran ZH. Prevalence and factors related to hepatitis $\mathrm{B}$ and $\mathrm{C}$ infection in inflammatory bowel disease patients in China: a retrospective study. J Crohns Colitis 2014;8:282-287.

54. Papa A, Felice C, Marzo M, et al. Prevalence and natural history of hepatitis $\mathrm{B}$ and $\mathrm{C}$ infections in a large population of IBD patients treated with anti-tumor necrosis factor-alpha agents. J Crohns Colitis 2013;7:113-119.

55. Brunasso AM, Puntoni M, Gulia A, Massone C. Safety of antitumour necrosis factor agents in patients with chronic hepatitis C infection: a systematic review. Rheumatology (Oxford) 2011;50:1700-1711.

56. Chinese Society of Hepatology; Chinese Medical Association, Wei L; Chinese Society of Infectious Diseases; Chinese Medical Association, Hou JL. The guideline of prevention and treatment for hepatitis C: a 2015 update. Zhonghua Gan Zang Bing Za Zhi 2015;23:906-923.

57. Leffler DA, Lamont JT. Clostridium difficile infection. N Engl J Med 2015;373:287-288.

58. Li Y, Qian JM. Identification and treatment strategies of inflammatory bowel disease with Clostridium difficile infection. Chin J Gastroenterol Hepatol 2016;25:1088-1090.

59. Xun J, Xia B, Peng M, Chen LP, Wang W, Yi FM. Relationship between inflammatory bowel disease and Clostridium difficile infection. Med J Wuhan Univ 2012;33:680-683.

60. Jin K, Wang S, Huang Z, Lu S. Clostridium difficile infections in China. J Biomed Res 2010;24:411-416.

61. Guo FL, Wen SB, Hu M, Zhang XQ. Comparison of five handhygiene methods for removal of Clostridium difficile. Chin J Disinfection 2015;32:737-738.

62. Luciano JA, Zuckerbraun BS. Clostridium difficile infection: prevention, treatment, and surgical management. Surg Clin North Am 2014;94:1335-1349. 
63. Schneeweiss S, Korzenik J, Solomon DH, Canning C, Lee J, Bressler B. Infliximab and other immunomodulating drugs in patients with inflammatory bowel disease and the risk of serious bacterial infections. Aliment Pharmacol Ther 2009;30:253264.

64. Trifan A, Stanciu C, Stoica O, Girleanu I, Cojocariu C. Impact of Clostridium difficile infection on inflammatory bowel disease outcome: a review. World J Gastroenterol 2014;20:1173611742.

65. Brown NA, Lebar WD, Young CL, Hankerd RE, Newton DW. Diagnosis of Clostridium difficile infection: comparison of four methods on specimens collected in Cary-Blair transport medium and tcdB PCR on fresh versus frozen samples. Infect Dis Rep 2011;3:e5. doi: 10.4081/idr.2011.e5.

66. Xu R, Ge P, Chen R, Liu XJ. Comparison of three different methods for detection of Clostridium difficile. Lab Med 2015; 30:201-205.

67. Owens RC. Clostridium difficile-associated disease: changing epidemiology and implications for management. Drugs 2007; 67:487-502.

68. McFarland LV, Elmer GW, Surawicz CM. Breaking the cycle: treatment strategies for 163 cases of recurrent Clostridium difficile disease. Am J Gastroenterol 2002;97:1769-1775.

69. Nelson RL, Kelsey P, Leeman H, et al. Antibiotic treatment for Clostridium difficile-associated diarrhea in adults. Cochrane Database Syst Rev 2011;(9):CD004610. doi: 10.1002/14651858.CD004610.pub4.

70. Drekonja DM, Butler M, MacDonald R, et al. Comparative effectiveness of Clostridium difficile treatments: a systematic review. Ann Intern Med 2011;155:839-847.

71. McDonald EG, Lee TC. Treatment for Clostridium difficile infection in adults. JAMA 2015;313:1974-1975.

72. Zar FA, Bakkanagari SR, Moorthi KM, Davis MB. A comparison of vancomycin and metronidazole for the treatment of Clostridium difficile-associated diarrhea, stratified by disease severity. Clin Infect Dis 2007;45:302-307.

73. Berg AM, Kelly CP, Farraye FA. Clostridium difficile infection in the inflammatory bowel disease patient. Inflamm Bowel Dis 2013;19:194-204.

74. Weiss K, Louie T, Miller MA, Mullane K, Crook DW, Gorbach SL. Effects of proton pump inhibitors and histamine-2 receptor antagonists on response to fidaxomicin or vancomycin in patients with Clostridium difficile-associated diarrhoea. BMJ Open Gastroenterol 2015;2:e000028. doi: 10.1136/bmjgast-2014-000028.

75. Issa M, Vijayapal A, Graham MB, et al. Impact of Clostridium difficile on inflammatory bowel disease. Clin Gastroenterol Hepatol 2007;5:345-351.
76. Ben-Horin S, Margalit M, Bossuyt P, et al. Combination immunomodulator and antibiotic treatment in patients with inflammatory bowel disease and clostridium difficile infection. Clin Gastroenterol Hepatol 2009;7:981-987.

77. Park DI, Hisamatsu T, Chen M, et al. Asian Organization for Crohn's and Colitis and Asia Pacific Association of Gastroenterology consensus on tuberculosis infection in patients with inflammatory bowel disease receiving anti-tumor necrosis factor treatment. Part 1: risk assessment. Intest Res 2018;16:4-16.

78. Jauregui-Amezaga A, Turon F, Ordás I, et al. Risk of developing tuberculosis under anti-TNF treatment despite latent infection screening. J Crohns Colitis 2013;7:208-212.

79. Abreu C, Magro F, Santos-Antunes J, et al. Tuberculosis in anti-TNF-alpha treated patients remains a problem in countries with an intermediate incidence: analysis of 25 patients matched with a control dpopulation. J Crohns Colitis 2013;7: e486-e492.

80. Van Assche G, Lewis JD, Lichtenstein GR, et al. The London position statement of the World Congress of Gastroenterology on Biological Therapy for IBD with the European Crohn's and Colitis Organisation: safety. Am J Gastroenterol 2011;106:15941602.

81. Dignass A, Van Assche G, Lindsay JO, et al. The second European evidence-based consensus on the diagnosis and management of Crohn's disease: current management. J Crohns Colitis 2010;4:28-62.

82. Expert group on the prevention and management of tuberculosis in the application of tumor necrosis factor antagonists. Expert consensus on the prevention and management of tuberculosis in the use of tumor necrosis factor antagonists. Chin J Rheumatol 2013;17:508-512.

83. Ohtsuki M, Terui T, Ozawa A, et al. Japanese guidance for use of biologics for psoriasis (the 2013 version). J Dermatol 2013; 40:683-695.

84. Kim HA, Yoo CD, Baek HJ, et al. Mycobacterium tuberculosis infection in a corticosteroid-treated rheumatic disease patient population. Clin Exp Rheumatol 1998;16:9-13.

85. Klein $\mathrm{NC}$, Go $\mathrm{CH}$, Cunha BA. Infections associated with steroid use. Infect Dis Clin North Am 2001;15:423-432.

86. Lai CC, Lee MT, Lee SH, Lee SH, Chang SS, Lee CC. Risk of incident active tuberculosis and use of corticosteroids. Int J Tuberc Lung Dis 2015;19:936-942.

87. Orlicka K, Barnes E, Culver EL. Prevention of infection caused by immunosuppressive drugs in gastroenterology. Ther Adv Chronic Dis 2013;4:167-185. 
88. Cardoso IP, de Almeida NP, Gotardo DR, Cardeal M, Santana GO. Tuberculin skin testing in inflammatory bowel disease patients from an endemic area of Brazil. Braz J Infect Dis 2014; 18:60-64.

89. Getahun H, Matteelli A, Abubakar I, et al. Management of latent Mycobacterium tuberculosis infection: WHO guidelines for low tuberculosis burden countries. Eur Respir J 2015;46: $1563-1576$

90. Shi XC, Zhang LF, Zhang YQ, Liu XQ, Fei GJ. Clinical and laboratory diagnosis of intestinal tuberculosis. Chin Med J (Engl) 2016;129:1330-1333.

91. Li J, Yang QT, Yue JR, et al. Evaluation of interferon-gamma release assays and tuberculin skin test in screening active tuberculosis and latent tuberculosis infection in close contacts of pulmonary tuberculosis patients. Chin J Antituberc 2015; 37:748-752.

92. Lin L, Chen YY, Wang SX. The clinical application value of interferon gamma release assays for diagnosis of latent tuberculosis infection in children. Zhejiang Med Educ 2014;13:54-56.

93. Zhao SY. Diagnosis and treatment of latent tuberculosis infection in children. Chin J Pract Pediatr 2003;18:395-397.

94. Zhang LF, Liu XQ. Advances in clinical applications of T-SPOT. TB for interferon-gamma release assay in the immunodiagnosis of tuberculosis. Zhongguo Yi Xue Ke Xue Yuan Xue Bao 2009;31:506-510.

95. Peng YL, Liu CY, Zhang HQ, Hou YP, Sun Q. The status of tuberculosis latent infection and analysis to laboratory monitoring indicators in high-risk medical staff. J Clin Pulm Med 2011; 16:546-547.

96. Chang KC, Leung CC. Systematic review of interferon-gamma release assays in tuberculosis: focus on likelihood ratios. Thorax 2010;65:271-276.

97. Amanatidou V, Syridou G, Mavrikou M, Tsolia MN. Latent tuberculosis infection in children: diagnostic approaches. Eur J Clin Microbiol Infect Dis 2012;31:1285-1294.

98. Chinese Medical Association Tuberculosis Branch; Chinese Journal of Tuberculosis and Respiratory Diseases Editorial Committee. Suggestion on the application of interferon-gamma release assays in China. Chin J Tuberc Respir Dis 2014;37: 744-747.

99. Park DI, Hisamatsu T, Chen M, et al. Asian Organization for Crohn's and Colitis and Asia Pacific Association of Gastroenterology consensus on tuberculosis infection in patients with inflammatory bowel disease receiving anti-tumor necrosis factor treatment. Part 2: management. Intest Res 2018;16:17-25.

100. Wu D, Yang H, Li Y, Li JN, Qian JM. Clinical features of pulmonary abnormalities associated with inflammatory bowel disease. Chin J Gastroenterol Hepatol 2016;25:1132-1135.
101. Niu HJ, Su BZ. Progress of fungi in gut and the related diseases. Chin J Mycol 2015;10:377-384.

102. Underhill DM, Iliev ID. The mycobiota: interactions between commensal fungi and the host immune system. Nat Rev Immunol 2014;14:405-416.

103. Che Y, Liu K, Li QR. The development of fungal community in intestinal diseases. Parenter Enteral Nutr 2015;22 378-381.

104. Li Q, Wang C, Tang C, He Q, Li N, Li J. Dysbiosis of gut fungal microbiota is associated with mucosal inflammation in Crohn's disease. J Clin Gastroenterol 2014;48:513-523.

105. Badiee P. Evaluation of human body fluids for the diagnosis of fungal infections. Biomed Res Int 2013;2013:698325. doi: $10.1155 / 2013 / 698325$.

106. Nawrot U, Kowalska-Krochmal B, Sulik-Tyszka B, et al. Evaluation of blood culture media for the detection of fungi. Eur J Clin Microbiol Infect Dis 2015;34:161-167.

107. Sands BE, Cuffari C, Katz J, et al. Guidelines for immunizations in patients with inflammatory bowel disease. Inflamm Bowel Dis 2004;10:677-692.

108. Rahier JF, Ben-Horin S, Chowers Y, et al. European evidencebased consensus on the prevention, diagnosis and management of opportunistic infections in inflammatory bowel disease. J Crohns Colitis 2009;3:47-91.

109. Wasan SK, Baker SE, Skolnik PR, Farraye FA. A practical guide to vaccinating the inflammatory bowel disease patient. Am J Gastroenterol 2010;105:1231-1238.

110. Loras C, Gisbert JP, Saro MC, et al. Impact of surveillance of hepatitis $b$ and hepatitis $c$ in patients with inflammatory bowel disease under anti-TNF therapies: multicenter prospective observational study (REPENTINA 3). J Crohns Colitis 2014;8: 1529-1538.

111. Gisbert JP, Villagrasa JR, Rodríguez-Nogueiras A, Chaparro $M$. Efficacy of hepatitis B vaccination and revaccination and factors impacting on response in patients with inflammatory bowel disease. Am J Gastroenterol 2012;107:1460-1466.

112. Mast EE, Weinbaum CM, Fiore AE, et al. A comprehensive immunization strategy to eliminate transmission of hepatitis B virus infection in the United States: recommendations of the Advisory Committee on Immunization Practices (ACIP) Part II: immunization of adults. MMWR Recomm Rep 2006;55:133.

113. WHO Publication. Hepatitis B vaccines: WHO position paper-recommendations. Vaccine 2010;28:589-590.

114. Are booster immunisations needed for lifelong hepatitis B immunity? European Consensus Group on Hepatitis B Immunity. Lancet 2000;355:561-565.

115. Lemon SM, Thomas DL. Vaccines to prevent viral hepatitis. N Engl J Med 1997;336:196-204. 
116. Morisco F, Castiglione F, Rispo A, et al. Effect of immunosuppressive therapy on patients with inflammatory bowel diseases and hepatitis B or C virus infection. J Viral Hepat 2013;20: 200-208.
117. Fiorino G, Peyrin-Biroulet L, Naccarato P, et al. Effects of immunosuppression on immune response to pneumococcal vaccine in inflammatory bowel disease: a prospective study. Inflamm Bowel Dis 2012;18:1042-1047. 